\title{
A Study of Word-Color Association for Pakistani and American Cultures
}

\author{
Ahsan Abdullah \\ Department of Computer Science, Air University, Multan Campus, Pakistan \\ E-mail: ahsan.abdullah@aumc.edu.pk
}

Received: December 16, 2020

Accepted: June 2, 2021 Published: July 2, 2021

doi:10.5296/ijssr.v9i2.18828

URL: https://doi.org/10.5296/ijssr.v9i2.18828

\begin{abstract}
Every region has its own culture, values and traditions that are unique for that region and that vary from region to region and country to country. These cultural differences are also reflected in the choice and association of color; concept word association is one of those differences. In this paper, we study the color related cultural similarities and dissimilarities between United States of America (USA) and Pakistan (PK). The study is based on 8 questions asked from 280 respondents (184 USA and $96 \mathrm{PK}$ ) for three concept words i.e., high quality, reliable, cheap and color disliked and liked with reference to 10 colors using the RGB model. After external data validation subsequent analysis showed high similarity of word-color association between respondents from USA and PK for the amber colors i.e., yellow, brown and orange for like-dislike and cheap-dislike but clear disparity for black (high quality) and blue (like-reliable). This information could be useful for multinational companies for tailoring their digital marketing campaigns accordingly.
\end{abstract}

Keywords: colors, Pakistan, USA, high-quality, reliable, cheap, dislike, dislike 


\section{Introduction}

\subsection{Background}

Undoubtly, colors do affect a person's feelings, mood, choices and subsequent activities. Although perception of colors can differ from person to person, but some colors' do have universal associations while other colors have cultural underpinnings. Some universal non-cultural aspects of colors are associated with the perception of optic nerves. For example, warm colors like red, orange and yellow require more optic nerves to sense them than cool colors that include blue, purple and green. And thus, warm colors evoke emotions like warmth, anger and hostility. In contrast, cool colors evoke emotions like calm, sadness and indifference and effect consumer decisions (Beta, 2020; Cherry, 2013; Posadas, 2009). Culture is a way of life of people. Traditionally people from the same culture belonging to same geography share same values and ideas. Some cultures carry meanings for some specific colors that are different from other cultures, such as the differences in color connotations' such as between the Western and Eastern cultures. For example, while black color in Western cultures indicates death, power, control while it indicates prosperity, wealth and health in certain Eastern cultures. Also, orange color is the color of affordability or cheap items in Western cultures, but it is the color of happiness and spirituality in certain Eastern cultures (Cultural Color, 2009).

\subsection{Statement of Problem}

Culture encompasses traditions and common norms, usually unwritten rules passed through and developed through generations. One such unwritten rule being association between words and colors. Colors are known to influence people's feelings and moods. In different cultures the choice of word-color associations vary depending on age, gender, culture, economy etc. The choice of color in marketing is known to have a significant impact on business. In this study we will analyze the results of word-color associations between respondents from Pakistan (PK) and USA for three words i.e., cheap, reliable, high quality and seven colors i.e., brown, green, blue, red, yellow, purple, and orange and three aromatic colors i.e., grey, black and white. Each color has a meaning which is different depending on the culture of a country. Comprehension of culture specific symbolism is important for any entity doing business with customers from other societies and cultures. Therefore, this study shows how the results can help marketers from USA (and vice-a-versa PK) to improve their business when they know the cultural effect of specific colors. Results of this study are based on data collected by distributing the color association survey as per published studies, such as Joe Hallock (http://www.joehallock.com), the results were gathered from an online survey of 96 respondents from Pakistan and 184 respondents from USA.

\section{Literature Review}

Colors, their meanings and their impact on the surrounding environment are scientifically interesting and is researched by many researchers and published in many scientific papers. There is an ongoing research to study the association between colors and emotional concept words. Strong relationships exist between colors and words or concepts. Interpretation of 
these relationships is referred to as color emotions, and is important for effective understanding of culture through use of color in design and art. Usually the associations have been methodically studied in experiments where subjects scale colors corresponding to bipolar attributes such as warm or cool.

In (Chen et al., 2020) a technique for studying the associations between words and colors is proposed and validated. A psychophysical experiment was defined where subjects select colors with reference to words. The data collected was used to demonstrate that many parallels do exist between the word-color associations for UK and Chinese subjects, yet some fascinating differences were also discovered. The technique makes unambiguous observation of non-existence of a one-to-one association among the words and colors. Researchers claim that their method can be applied to explore word-color associations for specific words and groups of participants or could be used to generate ground-truth data for assessing methods for programmatically producing the word-color associations.

In (Casas \& Chinoperekweyi, 2019) the researchers propose that color psychology is one of the main attributes that define the consumer behavior in the apparel market. They collected data through secondary sources such as articles, journals, and empirical sources such as, current researches concerning color psychology, consumer behavior concerning product color, and cultural acuities on color inside the apparel industry. By studying the color perception of different cultures, they concluded that employing a color linked with negative connotations will negatively affect the customers' retrieval indications, therefore deterring them from buying the product or service.

In (Hoppu et al., 2018) the importance of color perception of flavor and food selection was studied. The aim was to estimate the visual color opinion of liquid samples among Finnish adult buyers with reference to their background attributes. The subjects $(n=205)$ ranked six different colored solutions simply by looking for four attributes i.e., from most to least pleasant, healthy, sweet and sour. The color frequently regarded as the most pleasant was red $(37 \%)$, the healthiest was white $(57 \%)$, the sweetest red and orange (34\% both) and the sourest color being yellow (54\%). Assessments of certain colors varied across gender, age, body mass index (BMI) and education groups. Females considered red color as the sweetest more frequently than males $(p=0.013)$ while overweight subjects evaluated orange color as the sweetest more often than normal weight subjects $(p=0.029)$. Personalities may be connected with some disagreements in color associations

In (Sutton \& Altarriba, 2016) impact of colors on an assortment of human actions and behavior is studied, such as identification of facial expressions, object recognition, and the ability to classify stimuli as negative or positive. The researcher's objective was to provide a large database to support stimulus creation and selection. The results have shown that brightness is frequently linked with positive connotation words while darkness with negative connotation words. Furthermore, words such as anger and disaster seem to be characteristically associated with red color. The purpose of the study was to construct standards for positive and negative connotation and emotion-laden words and corresponding color relationships. Subjects were asked to present the first color that came to their mind from a set of $150+$ emotional items. The results showed that the color RED was mostly linked with 
negative connotations and emotion-laden words, while YELLOW and WHITE were linked with positive connotation and emotion-laden words, respectively.

In (Willard et al., 2008) it is shown that the colors are essential factor which influence the whole learning environment including student, teacher, and their performance. This study includes the influence of colors on people which differs from person to person according to their age, gender and culture and comparing with other attributes. The study also presents a dictionary that recommends the suitable colors for each purpose and type of activities related to the color classes. Finally, the paper studies the physiological response to colors.

In (Dadfar et al., 2003) a study is presented of the cultural differences between Saudi and Swedes using an 11-question questionnaire, but unlike our work they do not deal with colors or USA or concepts like cheap, reliable and high quality. According to the strong relation between words meaning and colors, a method for creation of a word-color association lexicons was introduced (Saif, 2011). Then, several experiments were conducted in order to measure how much the word-color associations are accurate.

The researchers (Ravi \& Rui, 2009) reconcile in their report the discrepancy between results of several researches and previous studies about the effect of blue and green colors over performance (Sauder School of Business, 2009) They provide a process of suggested evidence that the activation of alternative motivations facilitates the effect of color on performing cognitive tasks. They have made an effort in understanding the psychological side of effects of colors. They suppose several theories about effect of red and blue color and study each one of them while providing the final results and analysis.

In the work (Zammitto \& Veronica, 2005) colors definitions and meanings describe the physical and psychological process of how to visualize them. They identify several color systems and the differences between them. Also, they identify the meanings, connotations and emotions for each color. The final and significant section in the paper is a guide for using colors in different components in designing digital games. A report presented by The Color Association of the US focuses on color trends for American businesses especially the design trends (The Color Association of the US, 2005). Authors also discussed the color psychology for businesses.

\section{Method}

For our study we collected respondent's data using an online survey (http://bit.ly/10quLSe), for which a close-ended survey approach was used for the survey conducted for this paper by creating. The questioner by Joe Hallock (http://www.joehallock.com) was used for the survey conducted for this paper by developing an online version of that questionnaire. The questionnaire was subsequently distributed via social network sites i.e., Facebook, twitter and through WhatsApp. This study analyzes five questions out of 28 questions Hallock (http://www.joehallock.com) i.e., which colors are associated with concepts of cheap, reliable and high-quality.

Figure 1 gives a comparison of number of respondents in PK and USA with reference to their age-group. 


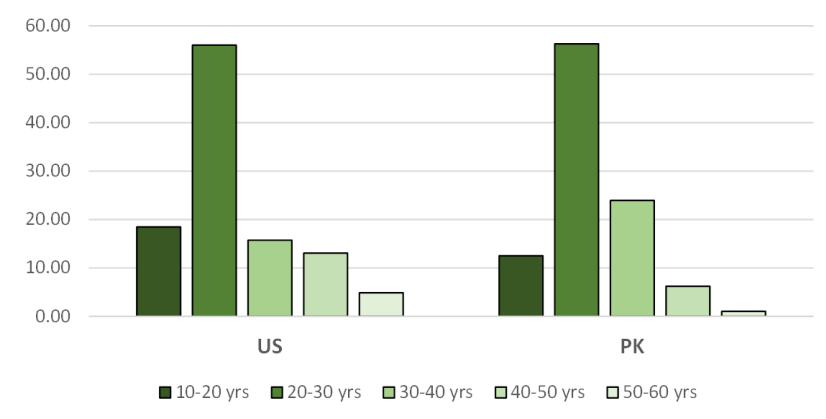

Figure 1. Comparison of PK and USA respondents' w.r.t Age

It can be observed that for both USA and PK, there is not much difference between the numbers of respondents in the age-group 20-30 years, but there are notable differences for the age-groups 10-20 years and 30-40 years, with PK at the higher end. While for the senior age group of 50-60 years the number of USA respondents is significantly higher as compared to the PK respondents.

Figure 2 gives a comparison of gender of respondents in PK and USA. It can be observed that as compared to PK there are more female respondents from USA. This is not surprising, because as per statista.com as of February 2019 females accounted for $78 \%$ of adults in the USA who use social networks as compared to $65 \%$ males.

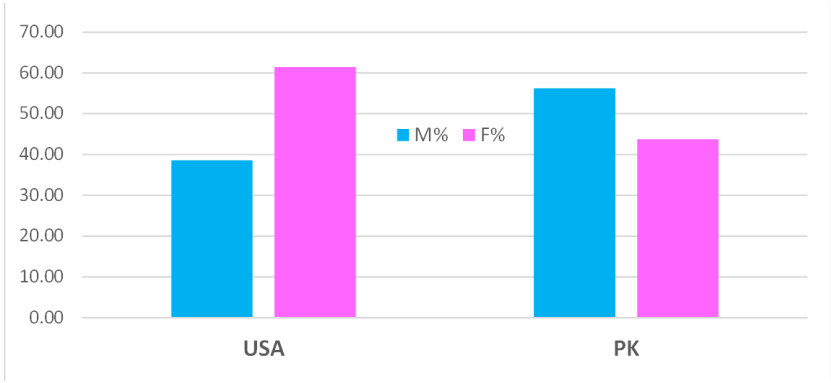

Figure 2. Comparison of PK and USA respondents' w.r.t gender

The Method section describes in detail how the study was conducted, including conceptual and operational definitions of the variables used in the study, Different types of studies will rely on different methodologies; however, a complete description of the methods used enables the reader to evaluate the appropriateness of your methods and the reliability and the validity of your results, It also permits experienced investigators to replicate the study, If your manuscript is an update of an ongoing or earlier study and the method has been published in detail elsewhere, you may refer the reader to that source and simply give a brief synopsis of the method in this section. 


\section{Macrothink}

\section{Results}

In this section we will present a graphical comparison of word-color association results between PK and USA respondents for the five questions and 10 colors. The USA respondent's response collected from Joe Hallock (http://www.joehallock.com).

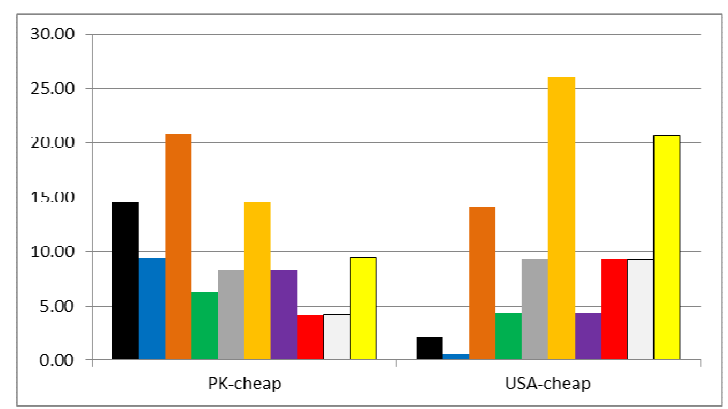

Figure 3. Color-comparison of USA and PK respondents for Cheap

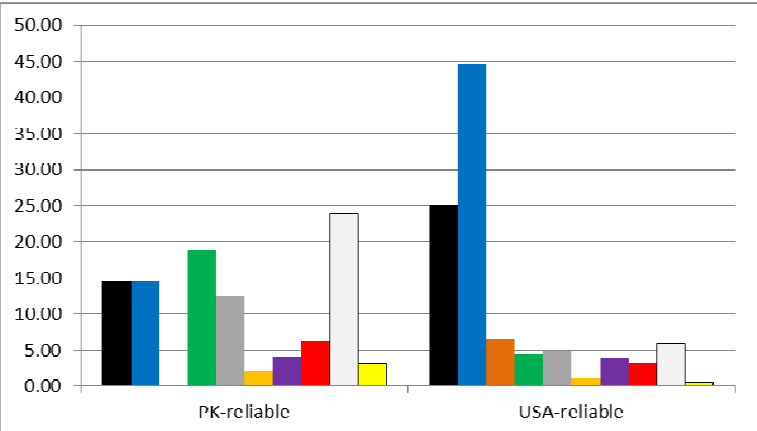

Figure 4. Color-comparison of USA and PK respondents for Reliable

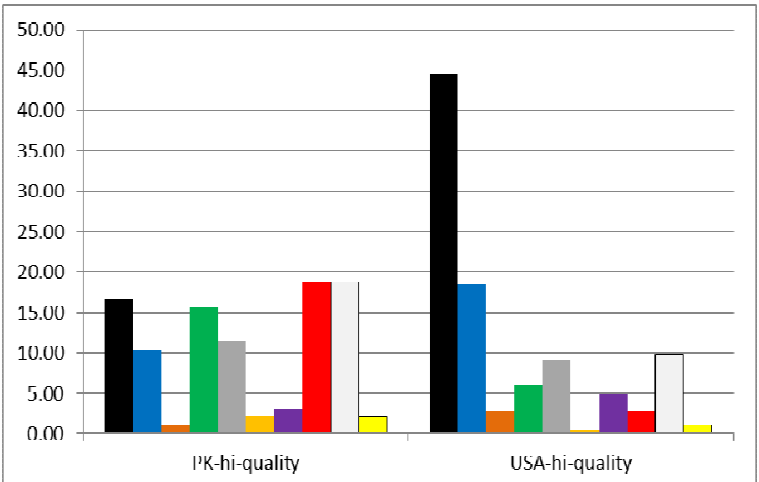

Figure 5. Color-comparison of USA and PK respondents for Hi-Quality 


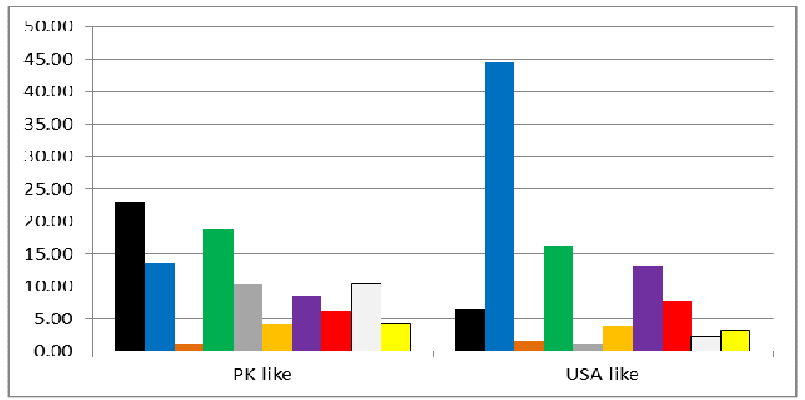

Figure 6. Color-comparison of USA and PK respondents for Like

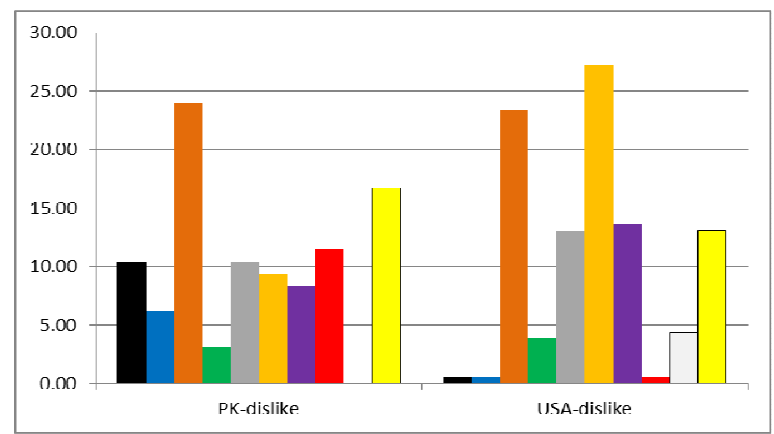

Figure 7. Color-comparison of USA and PK respondents for Dislike

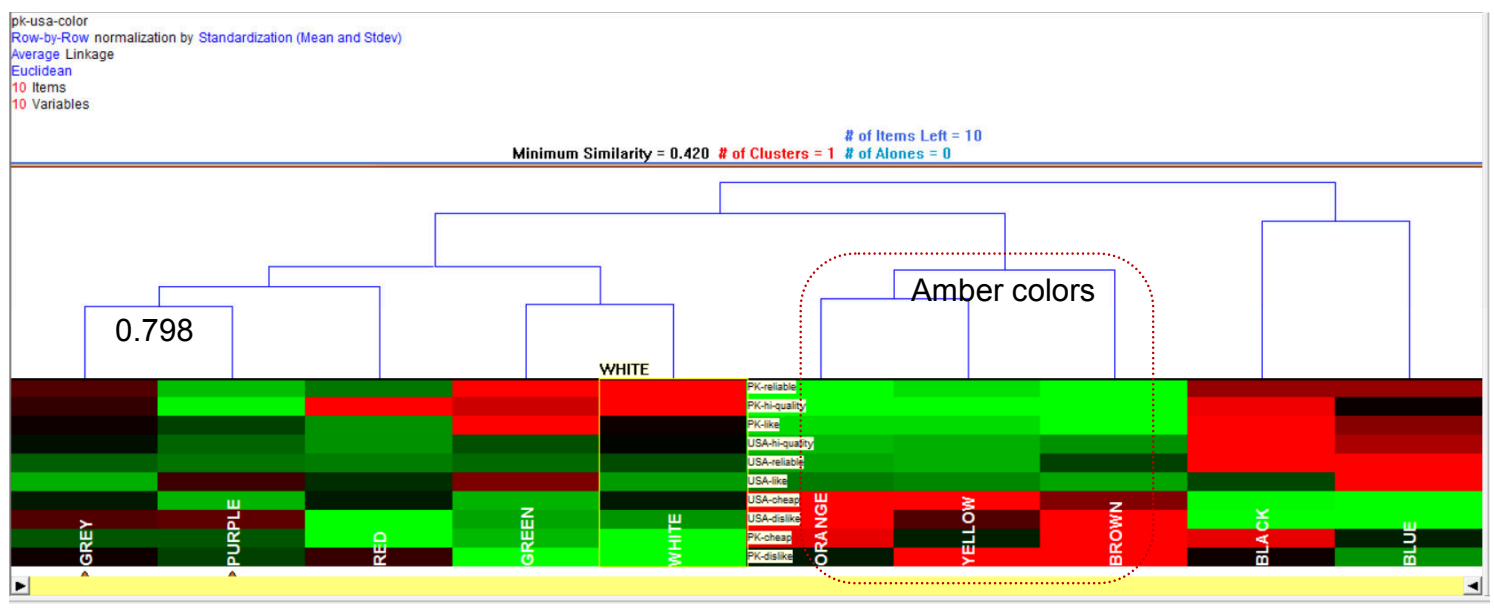

Figure 8. Clustering of Amber colors for USA and PK respondents

Finally, in this section we test the pair-wise color association for (Cheap, Reliable, High-Quality, Like and Dislike) by calculating the Pearson's correlation between the numbers of participants who assigned specific color for the pair of concept words considered in this paper. Due to scarcity of space, we will only consider the results for the PK respondents, as shown in Table 1. 
Table 1. Pair-wise correlation of word-color association for five questions

\begin{tabular}{llllll}
\hline & cheap & reliable & hi-quality & like & dislike \\
\hline cheap & 1 & -0.552 & -0.613 & -0.23 & 0.713 \\
reliable & -0.552 & 1 & 0.797 & 0.727 & -0.805 \\
hi-quality & -0.613 & 0.797 & 1 & 0.647 & -0.615 \\
like & -0.23 & 0.727 & 0.647 & 1 & -0.568 \\
dislike & 0.713 & -0.805 & -0.615 & -0.568 & 1 \\
\hline
\end{tabular}

\section{Discussion}

From Figure 4 a major difference can be observed for the choice of color associated with concept word reliable i.e., blue for USA and white for PK. When the American flag was adopted in 1777, there were no meanings associated with the three colors in the flag i.e., red, white and blue. However, subsequently the colors in the Great Seal of USA did have specific meanings associated. It was stated by the Secretary of the Continental Congress that "Blue, the color of the Chief (the broad band above the stripes) signifies vigilance, perseverance \& justice" http://www.usflag.org/colors.html. This explains the significance of blue for USA both in reliability and being the liked color. In PK men wear loose white clothing kurta during summer; the color is very important, as white color reflects sunlight and keeps the person cool in the sunlight. Furthermore, white is also associated with piousness with religious connotations. Thus, in PK and other parts of the region one could trust on white color for being protecting from sunlight and sins. However, in the PK flag, white color is associated with minorities.

From Figure 5 a major difference in the choice of color for high quality can be observed i.e., black for USA and white-red for PK. The significance of white we have already discussed for PK. Traditionally the bridal dress worn by the bride on the day of wedding is of red color and most of the cases the bride is a virgin (first marriage i.e., not a divorcee or a widow) signifying piousness which translates into high quality connotations.

From Figure 3 and Figure 7 it can be observed without doubt that brown is the color associated with cheapness both in PK and USA, however, color orange was not disliked by any respondents from PK. To explore this future, we performed hierarchical clustering of responses for the word-color associations for the respondents from PK and USA with the results shown in Figure 8. It can be observed that the Amber colors i.e., Orange, Yellow and Brown have clustered together for the two cultures that are thousands of miles apart. To collectively identify the relationship for the PK results shown in Figure 3 to Figure 7, we create a pair-wise similarity matrix using Pearson's' correlation. The results of that matrix are shown in Table 1 where significant positive correlation i.e., $>0.5$ shown by green color and significant negative correlation i.e., $<-0.5$ shown by red color. From Table 1 it can be observed that dislike is negatively correlated with reliable and high-quality, and positively correlated with the word dislike. This is understandable as these two words (and corresponding colors) are related to serious matters. The highest correlation i.e., 0.797 is 
between quality and reliability. However, an interesting question is, if the word-color association for the word cheap is based on their perceived association or based on the corresponding color being disliked? The answer to this question could be part of future work.

\section{Conclusion}

Although USA and PK are countries that are geographically far apart, yet based on the analysis of the choice of colors for cheap and dislike and their correlation the two cultures seem to be more similar than different. Tis similarity, could be due to the shrinking of the physical distances due to internet and satellite TV resulting in the world becoming a global village. The differences in word-color association for black and blue could be deep-rooted with historical underpinnings.

\section{References}

Beta, V. (2020). Color Psychology on the Packaging and how it affects the purchase.

Casas, M. C., \& Chinoperekweyi, J. (2019). Color psychology and its influence on consumer buying behavior: A case of apparel products. Saudi Journal of Business and Management Studies, 4(5), 441-456.

Chen, Y., Yang, J., Pan, Q., Vazirian, M., \& Westland, S., (2020). A method for exploring word-colour associations. Color Research \& Application, 45(1), 85-94. https://doi.org/10.1002/col.22434

Cherry, K (2013). Color Psychology. Psychology. Retrieved from http://psychology.about.com/od/sensationandperception/a/colorpsych.htm

Cultural Color. (2009). Empower-yourself-with-color-psychology. Retrieved from http://www.empower-yourself-with-color-psychology.com/cultural-color.html

Dadfar, A. et al. (2003). Intercultural aspects of doing business with Saudi Arabia. Linkoping University, Linkoping.

Daggett, W., Cobble, J., \& Gertel, S. (2008). Color in an Optimum Learning Environment, International Center for Leadership in Education. March, 2008.

Gerard, R. M. (1976). Differential effects of colored lights on psychophysiological functions. $\mathrm{PhD}$ diss., University of California, Los Angeles.

Hoppu, U., Puputti, S., Aisala, H., Laaksonen, O., \& Sandell, M. (2018). Individual differences in the perception of color solutions. Foods, 7(9), 154. https://doi.org/10.3390/foods7090154

Joe H. (n.d.). Color assignment. Retrieved from http://www.joehallock.com/edu/COM498/associations.html

Mohammad, S. (2011). Colorful language: Measuring word-color associations (pp. 97-106). In Proceedings of the 2nd Workshop on Cognitive Modeling and Computational Linguistics, Portland, Oregon. 


\section{Macrothink}

International Journal of Social Science Research

ISSN 2327-5510 2021, Vol. 9, No. 2

Morris, W. (2000). The American heritage dictionary of the English language. Boston: Houghton Mifflin.

Ravi, M., \& Rui, J. Z. (2009). Blue or red? Exploring the effect of color on cognitive task performances. Science, 323(5918), 1226-1229. https://doi.org/10.1126/science.1169144

Sylvia, P. (2009). The Meaning of Colors. Sibagraphics. Retrieved from http://www.sibagraphics.com/color.php

The Color Association of the US. (2005). Color Trends in the Corporate World.

Zammitto, V. (2005). The Expressions of Colors. In Conference Proceedings of DiGRA'05, Changing Views: Worlds in Play, Vancouver, Canada.

\section{Copyrights}

Copyright for this article is retained by the author(s), with first publication rights granted to the journal.

This is an open-access article distributed under the terms and conditions of the Creative Commons Attribution license (http://creativecommons.org/licenses/by/4.0/). 